\title{
Estimated glomerular filtration rate in patients overdosed with gentamicin
}

https://doi.org/10.1515/labmed-2019-0124

Received July 24, 2019; accepted October 11, 2019; previously published online November 9, 2019

\begin{abstract}
Background: The aim of this study was to compare the estimated glomerular filtration rate (eGFR) from serum creatinine (eGFRcrea) and cystatin C (eGFRcys) in patients with elevated serum trough levels of gentamicin before the next planned dose during treatment in the intensive care unit (ICU).
\end{abstract}

Methods: This was a retrospective observational study. Patients who stayed in an ICU, received a once-daily dose of gentamicin, and who had results from all serum gentamicin trough levels, eGFRcrea and eGFRcys analyses were included in the study. Overdosed patients were defined as those with serum gentamicin trough levels above $1 \mathrm{mg} / \mathrm{L}$ before the next dose. Gentamicin was measured by a particle-enhanced turbidimetric immunoassay (PETIA). Creatinine and cystatin $\mathrm{C}$ were measured by standardized methods.

Results: The median age (range) was lower in all patients with gentamicin concentration measurements than in overdosed patients (67 [19-96] vs. 75 [48-99] years, respectively; $\mathrm{p}<0.0001$ ). The median interquartile range (IQR) of the eGFRcrea was higher than that of the eGFRcys in overdosed patients (60 [44-79] $\mathrm{mL} / \mathrm{min} / 1.73 \mathrm{~m}^{2}$ vs. 41 [29-58] $\mathrm{mL} / \mathrm{min} / 1.73 \mathrm{~m}^{2}$, respectively; $\left.\mathrm{p}<0.0001\right)$. The median IQR of the eGFRcrea was higher than that of the eGFRcys in controls (87 [78-98] mL/min/1.73 $\mathrm{m}^{2}$ vs. 66 [54-93] mL/ $\min / 1.73 \mathrm{~m}^{2}$, respectively; $\left.\mathrm{p}<0.0001\right)$.

\footnotetext{
*Correspondence: Assist. Prof. Tomáš Šálek, MD, PhD, Department of Clinical Biochemistry and Pharmacology, Tomas Bata Hospital, Havlíčkovo nábřeží 600, 76275 Zlín, Czech Republic; and Department of Biomedical Sciences, Medical Faculty of the University of Ostrava, Syllabova 19, 70300 Ostrava - Zábřeh, Czech Republic, Phone:+420602642663, E-Mail: tsalek@seznam.cz Martin Vodička: Department of Pharmacy, Tomas Bata Hospital, Zlín, Czech Republic

Tomáš Gabrhelík: Department of Anaesthesiology and Resuscitation, Tomas Bata Hospital, Zlín, Czech Republic
}

Conclusions: Overdosed patients had both a lower eGFRcrea and eGFRcys than controls. Elderly patients are the most commonly overdosed patients. We recommend measuring cystatin $\mathrm{C}$ and calculating the eGFRcys and combined equation (eGFRcrea + cys) in ICU patients over 65 years of age, which would enable improved gentamicin dosage adjustments.

Keywords: creatinine; cystatin C; estimated glomerular filtration rate; gentamicin; glomerular filtration.

Brief summary: Estimations of the glomerular filtration rate (GFR) from cystatin $C$ are much lower than those from creatinine in both patients overdosed with gentamicin and controls in intensive care units (ICUs). Elderly patients are the most commonly overdosed patients. We recommend to measure cystatin $C$ and calculate the estimated GFR of cystatin (eGFRcys) in patients in the ICU over 65 years of age receiving gentamicin, which would enable better consideration of the dose.

\section{Introduction}

Gentamicin is a bactericidal antibiotic used in the treatment of Gram-negative infections in intensive care units (ICUs). Gentamicin causes an irreversible inhibition of protein synthesis on ribosomes. This drug belongs to the aminoglycoside group of antibiotics, which have a concentration-dependent killing effect. This means that a higher concentration of gentamicin kills a higher proportion of bacteria. Gentamicin also has a post-antibiotic effect in treating patients with Gram-negative infections. This means that bacteria are also killed when the plasma concentration of the antibiotic is below the minimum inhibitory concentration. This effect enables once-daily dosing [1].

Gentamicin is mainly administered as a 30- to 60-min infusion because it is poorly absorbed from the gastrointestinal tract. This compound is highly polar and has a low volume of distribution. The half-life of gentamicin in plasma is $2-3 \mathrm{~h}$, but the half-life can be extended up to $48 \mathrm{~h}$ in patients with a decreased glomerular filtration 
rate (GFR). Serum gentamicin concentrations and gentamicin clearance are the best parameters for gentamicin dose adjustments [2]. Knowing the GFR is therefore of great importance for dosing if rapid gentamicin level measurements are not available. According to the Kidney Disease Improving Global Outcomes (KDIGO) guidelines, the dose should be reduced if the GFR is below $60 \mathrm{~mL} /$ $\min [3]$.

The estimated glomerular filtration rate from serum creatinine (eGFRcrea) is usually used as a marker of the GFR in clinical practice, but the concentration of serum creatinine depends on muscle mass. Patients in ICUs often lose muscle mass [4]. The serum creatinine concentration of these patients might be falsely low, and the eGFRcrea may be falsely high. Critical illness is associated with significant decreases in serum creatinine that persist until hospital discharge, potentially causing an inaccurate assessment of renal function [5].

Since 2012, the standardized measurement of cystatin $\mathrm{C}$ has been available in clinical practice. Cystatin $\mathrm{C}$ is an inhibitor of cysteine proteases. This molecule is produced by all nucleated cells of the body at a constant rate. Serum cystatin $\mathrm{C}$ does not depend on muscle mass [6].

Overdosing with gentamicin can cause nephrotoxic and ototoxic side effects. These side effects are associated with an increased trough concentration. The safety limit of the trough concentration is below $1 \mathrm{mg} / \mathrm{L}$ for oncedaily dosing [7]. The therapeutic drug monitoring (TDM) of gentamicin is recommended to reduce the number of side effects [8].

Appropriate drug dosing is very important for patient safety. Muscle mass reduction in patients in ICUs is significant. Therefore, we analyzed the differences between the eGFRcrea and eGFRcys in patients overdosed with gentamicin and in controls to find the best estimation of the GFR for gentamicin dosing when rapid gentamicin level measurements are not available.

\section{Materials and methods}

\section{Subjects}

This was a retrospective observational study. The study lasted from September 2015 to September 2018. Patients who had stayed in an ICU with once-daily gentamicin dosing and who had all the results of serum gentamicin trough levels, eGFRcrea and eGFRcys analyses were included in the study. Dobutamine treatment was the exclusion criterion. Overdosed patients were defined by serum gentamicin trough levels above $1 \mathrm{mg} / \mathrm{L}$ before the next dose.

Patients who received $240 \mathrm{mg}$ of gentamicin once daily and had serum gentamicin through levels below $0.5 \mathrm{mg} / \mathrm{L}$ (below the limit of quantification of the laboratory method with a coefficient of variation of $\leq 20 \%$ ) were regarded as not overdosed. From the non-overdosed patients, the oldest patients were selected to form the control group, which had the same number of members as the overdosed group. Nevertheless, the median age of the overdosed patients was higher than that of the control group.

\section{The TDM approach}

The once-daily approach for gentamicin (aminoglycoside) dosing was used at our hospital. When the trough concentration was above $1.0 \mathrm{mg} / \mathrm{L}$, we usually prolonged the dosing interval.

For all TDM requests, we routinely used an electronic request form that includes the drug concentration, first day of administration, time of the last dose, dosing interval, dose, route of administration and duration of infusion.

The laboratory report contained the drug concentration and an interpretative comment that recommended the quantity of the next dose or the extension of the dosing interval. This interpretative comment was made by a specialized clinical pharmacologist or a pharmacist.

\section{Laboratory tests and estimations of the GFR}

Gentamicin concentrations were measured by the particle-enhanced turbidimetric immunoassay (PETIA) method. Creatinine was measured by the sarcosine oxidase-based enzymatic standardized method traceable to the certified reference material NIST SRM 967 [9]. Cystatin $\mathrm{C}$ was measured by the PETIA method traceable to the certified reference material DA ERM 471 [10]. The estimations of GFRs were calculated according to the Chronic Kidney Disease Epidemiology Collaboration (CKD-EPI) equations, which were reported in 2009 for serum creatinine and in 2012 for serum cystatin [11]. All tests were performed on an Abbott Architect analyzer ci 16200 (Abbott Laboratories, Abbott Park, IL, USA).

All tests were available $168 \mathrm{~h}$ a week, and the ordering physicians knew the results immediately. Each result that provided a drug concentration was accompanied by an interpretative comment. 


\section{Statistical analysis}

The MedCalc statistical software version 17.4 (MedCalc Software bvba, Ostend, Belgium) was used for statistical analysis. Results with a p-value $<0.05$ were considered statistically significant.

The D'Agostino-Pearson test was used for assessment of the normality of eGFR results. The eGFR results did not have a normal distribution. Thus, nonparametric tests were used for analysis. The Mann-Whitney test for independent samples and the Wilcoxon test for paired samples were used for the comparison of medians. Spearman's rank correlation coefficient was used for correlation analysis. Bland-Altman plots were used for the comparison of pairs of eGFRs.

\section{Ethical approval}

This study was approved by the Ethics Committee of The Tomas Bata Hospital in Zlin, Czech Republic. This study was conducted in accordance with the Declaration of Helsinki. Informed consent was obtained from all individuals included in this study. Informed consent for the publication of anonymous patient data is part of the hospital stay consent. Patients can select yes or no.

\section{Results}

The serum gentamicin concentration was measured in 1010 patients. Cystatin C was ordered in 321 selected patients from the ICU for whom overdosing could occur. A total of 45 patients were overdosed, and the control group also had 45 patients. The overdosed group consisted of 23 males and 22 females.

The diagnoses of the overdosed patients varied. Of these patients, 25 suffered from type 2 diabetes mellitus. A total of 24 patients underwent surgery. There were polytraumatic patients and patients with pneumonia and sepsis. A total of 11 patients (24\%) among the overdosed patients died of sepsis. Of these patients, seven had type 2 diabetes mellitus.

The median age (range) of all patients with measured gentamicin concentrations was lower than that of overdosed patients (67 [19-96] vs. 75 [48-99] years, respectively; $\mathrm{p}<0.0001)$.

The median age (range) of controls was 70 (58-82) years. The pharmacokinetic parameters of the overdosed patients are displayed in Table 1. The median interquartile
Table 1: The pharmacokinetic parameters of overdosed patients.

\begin{tabular}{ll}
\hline Variable & Results \\
\hline Body weight median (IQR), kg & $80(70-87)$ \\
Gentamicin dose, mg & $80 \mathrm{mg}$ in 1 patient \\
& $160 \mathrm{mg}$ in 4 patients \\
& $240 \mathrm{mg}$ in 39 patients \\
& $320 \mathrm{mg}$ in 1 patient \\
Dose interval & $24 \mathrm{~h}$ \\
Predose gentamicin concentration & $2.0(1.6-2.6)$ \\
median (IQR), mg/L & \\
Predose gentamicin concentration & $1.3-3.4$ \\
(range), mg/L & 1 Dose in 23 patients \\
Number of doses before & 2 Doses in 7 patients \\
overdosing & 3 Doses in 6 patients \\
& 4 Doses in 5 patients \\
& 5 Doses in 1 patient \\
& 6 Doses in 1 patient \\
& 8 Doses in 1 patient \\
& 9 Doses in 1 patient \\
\hline
\end{tabular}

$I Q R$, interquartile range.

range (IQR) of the eGFRcrea and eGFRcys in overdosed patients and controls are shown in Table 2.

The Spearman rank correlation coefficient between the eGFRcrea and eGFRcys in overdosed patients was 0.762 $(p<0.0001)$. The Spearman rank correlation coefficient between the serum trough gentamicin concentrations and doses in overdosed patients was $-0.152(p=0.319)$. The Spearman rank correlation coefficient between the serum trough gentamicin concentrations and the number of previous doses in overdosed patients was $-0.281(p=0.062)$. The differences between pairs of eGFRs in overdosed patients are displayed in the Bland-Altman plot (Figure 1).

\section{Discussion}

In this study, we compared the eGFRcrea and eGFRcys in patients with increased predose levels of serum gentamicin and in controls.

Lipcsey et al. reported significant differences between the creatinine and cystatin C GFR estimations in the ICU. The eGFRcrea was higher than the eGFRcys [12]. This finding supports our results, which showed that the median eGFRcrea was higher than the median of eGFRcys in overdosed patients and in controls in the ICU.

Iversen et al. reported significant discrepancies in the eGFR and CKD classification when switching between the CKD-EPI eGFR equations in acutely hospitalized elderly patients. Switching from a creatinine-based equation to a 
Table 2: The medians (IQR) of the eGFRcrea and eGFRcys in overdosed patients and controls.

\begin{tabular}{llll}
\hline & eGFRcrea ${ }^{\mathrm{a}}, \mathrm{mL} / \mathrm{min} / \mathbf{1 . 7 3} \mathrm{m}^{2}$ & eGFRcys $^{\mathrm{b}}, \mathrm{mL} / \mathrm{min} / \mathbf{1 . 7 3} \mathrm{m}^{2}$ & $\mathrm{p}$-Value \\
\hline Overdosed $(\mathrm{n}=45)$ & $60(44-79)$ & $41(29-58)$ & $(\mathrm{p}<0.0001)$ \\
Controls $(\mathrm{n}=45)$ & $87(78-98)$ & $66(54-93)$ & $(\mathrm{p}<0.0001)$ \\
$\mathrm{p}$-Value & $(\mathrm{p}<0.0001)$ & $(\mathrm{p}<0.0001)$ & \\
\hline
\end{tabular}

aEstimated glomerular filtration rate from serum creatinine. ${ }^{\mathrm{b}}$ Estimated glomerular filtration rate from serum cystatin C. IQR, interquartile range.

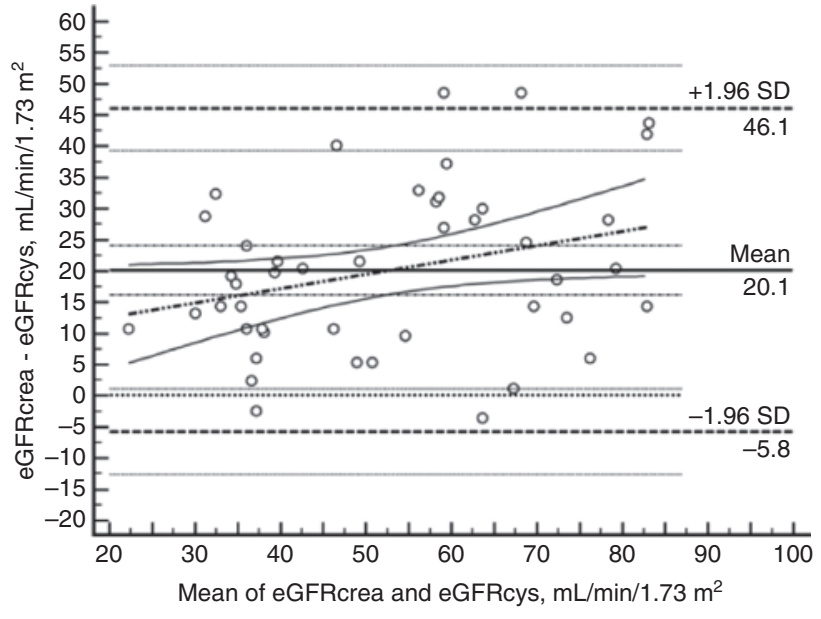

Figure 1: Bland-Altman plot: comparison of the eGFRcrea and eGFRcys in overdosed patients.

eGFRcrea, estimated glomerular filtration rate from serum creatinine; eGFRcys, estimated glomerular filtration rate from serum cystatin C; SD, standard deviation.

corresponding cystatin C-based equation resulted in lower GFR estimates, and these differences were larger in these patients than in community-dwelling older populations [13]. This observation is in accordance with our results.

The dose of gentamicin should be adjusted according to the gentamicin concentration. A reliable method for determining the GFR is needed in clinical practice if gentamicin concentration measurements are not available. Knowledge regarding the GFR is needed for initial dosing until gentamicin concentration measurements are available.

It is not easy to obtain the true GFR in clinical practice in the ICU. The reference (gold standard) method for determining the GFR is inulin clearance. This method is not available in routine clinical practice because it is difficult to perform and is time-consuming [14].

The clearance of creatinine was used as an indicator of the GFR for many years, but this test overestimates the true GFR [15]. The KDIGO guidelines recommend using the eGFRcys when the eGFRcrea may be inaccurate [16]. Muscle wasting is common in ICU patients.

A low GFR is also associated with a decrease in muscle mass. This decrease in muscle mass results in an overestimation of the eGFRcrea compared to the eGFRcys in patients with CKD and a low GFR [17]. In our ICU patients, the eGFRcys was lower than the eGFRcrea over the whole eGFR range. These results may also be explained by muscle wasting.

A case study by Rebollo and Cepeda-Piorno clearly described the use of cystatin $\mathrm{C}$ in a patient treated with another aminoglycoside antibiotic, amikacin. Serum aminoglycoside antibiotic concentrations also reflect the GFR but are less dependent on muscle mass [18]. This observation is consistent with our results.

Šálek and Adamíková reported a higher eGFRcrea than eGFRcys in elderly type 2 diabetic patients. This difference increased with increasing age [19]. The cohort of gentamicin-overdosed patients in the ICU also included elderly patients.

Having serum creatinine, cystatin $\mathrm{C}$ and gentamicin measurements available $168 \mathrm{~h}$ a week may also improve patient safety. The implementation of routine eGFRcys measurements in ICU patients receiving gentamicin may reduce overdosing, especially when rapid gentamicin level measurements are not available.

Cystatin C is also not an ideal marker for GFR. Stevens et al. reported that factors other than glomerular filtration affect serum cystatin $C$ concentrations. These factors include $\mathrm{C}$-reactive protein, diabetes, body mass index, etc. [20]. This means that cystatin C may be falsely elevated in ICU patients with acute phase reactions. The extent of this elevation is impossible to predict.

A study by Chin et al. recommended the combined CKD-EPI equation for the estimation of gentamicin clearance, which calculates the eGFR from both serum creatinine and cystatin $C$ [21]. The combined equation, which includes both creatinine and cystatin $\mathrm{C}$, may be the best solution because it may limit the disadvantages of either creatinine or cystatin C. All possible interfering conditions for both creatinine and cystatin $\mathrm{C}$ measurements should be considered in a particular patient before gentamicin dose adjustment. We also routinely calculate the combined CKD-EPI equation. The purpose of this study was to show the differences between the eGFRcrea and eGFRcys. All data can be found in the Supplementary Material. 
The major limitation of our study was that we did not measure the GFR with a reference method. This means that we do not know the true GFR, but this situation reflects real clinical practice with the high probability of muscle wasting in the acute care setting, which compromises the usefulness of the eGFRcrea.

The CKD-EPI equations were developed for hemodynamically stable patients. GFR estimations in the acute care setting have not been established.

Patients in the control group were younger than patients in the overdosed group.

The last limitation was the relatively small number of patients, but it is not easy to find patients with increased trough levels of gentamicin.

\section{Conclusions}

In summary, patients overdosed with gentamicin had a lower eGFRcrea and eGFRcys than controls. Elderly patients are the most commonly overdosed patients. We recommend measuring cystatin $\mathrm{C}$ and calculating the eGFRcys and eGFRcrea + cys in ICU patients over 65 years of age receiving gentamicin, which would enable better information for dosing mainly if serum gentamicin concentration results are not available. This practice would improve patient safety.

Author contributions: All the authors have accepted responsibility for the entire content of this submitted manuscript and approved submission.

Research funding: None declared.

Employment or leadership: None declared. Honorarium: None declared.

Competing interests: The funding organization(s) played no role in the study design; in the collection, analysis, and interpretation of data; in the writing of the report; or in the decision to submit the report for publication.

\section{References}

1. Gonzalez LS, Spencer JP. Aminoglycosides: a practical review. Am Fam Physician 1998;58:1811-20.

2. Kacî́ová I, Grundmann M. [Therapeutic monitoring of amikacin and gentamicin in routine clinical practice]. Vnitr Lek 2015;61:33-41.

3. Kidney Disease I Improving Global Outcomes. Chapter 4: Other complications of CKD: CVD, medication dosage, patient safety, infections, hospitalizations, and caveats for investigating complications of CKD. Kidney Int Suppl 2013;3:91-111.

4. Schefold JC, Bierbrauer J, Weber-Carstens S. Intensive care unitacquired weakness (ICUAW) and muscle wasting in critically ill patients with severe sepsis and septic shock. J Cachexia Sarcopenia Muscle 2010;1:147-57.

5. Prowle JR, Kolic I, Purdell-Lewis J, Taylor R, Pearse RM, Kirwan CJ. Serum creatinine changes associated with critical illness and detection of persistent renal dysfunction after AKI. Clin J Am Soc Nephrol 2014;9:1015-23.

6. Shlipak MG, Mattes MD, Peralta CA. Update on cystatin C: incorporation into clinical practice. Am J Kidney Dis 2013;62:595-603.

7. Deck DH, Winston LG. Aminoglycosides and spectinomycin. In: Katzung BG, editor. Basic and clinical pharmacology 13E. New York: McGraw-Hill Education, 2015:802.

8. Habib G, Lancellotti P, Antunes MJ, Bongiorni MG, Casalta J-P, Zotti Del F, et al. 2015 ESC Guidelines for the management of infective endocarditis. Eur Heart J 2015;36:3075-128.

9. Dodder NG, Tai S-C, Sniegoski LT, Zhang NF, Welch MJ. Certification of creatinine in a human serum reference material by GC-MS and LC-MS. Clin Chem 2007;53:1694-9.

10. Grubb A, Blirup-Jensen S, Lindström V, Schmidt C, Althaus $\mathrm{H}$, Zegers I, et al. First certified reference material for cystatin C in human serum ERM-DA471/IFCC. Clin Chem Lab Med 2010;48:1619-21.

11. Inker LA, Schmid CH, Tighiouart H, Eckfeldt JH, Feldman HI, Greene T, et al. Estimating glomerular filtration rate from serum creatinine and cystatin C. N Engl J Med 2012;367:20-9.

12. Lipcsey M, Furebring M, Rubertsson S, Larsson A. Significant differences when using creatinine, modification of diet in renal disease, or cystatin C for estimating glomerular filtration rate in ICU patients. Ups J Med Sc 2011;116:39-46.

13. Iversen E, Bodilsen AC, Klausen H, Treldal C, Andersen O, Houlind $M$, et al. Kidney function estimates using cystatin $C$ versus creatinine: impact on medication prescribing in acutely hospitalized elderly patients. Basic Clin Pharmacol Toxicol 2019;124:466-78.

14. Soveri I, Berg UB, Björk J, Elinder C-G, Grubb A, Mejare I, et al. Measuring GFR: a systematic review. Am J Kidney Dis 2014;64:411-24.

15. Jabor A, editor. Internal environment. Praha: Grada Publishing a.s., 2008.

16. Kidney Disease | Improving Global Outcomes. Chapter 1: definition and classification of CKD. Kidney Int Suppl 2013;3:19-62.

17. Sharma D, Hawkins M, Abramowitz MK. Association of sarcopenia with eGFR and misclassification of obesity in adults with CKD in the United States. Clin J Am Soc Nephrol 2014;9:2079-88.

18. Rebollo N, Cepeda-Piorno FJ. Cystatin C for therapeutic drug monitoring. Clin Chem 2015;61:804-7.

19. Šálek T, Adamíková A. Cystatin C measurement leads to lower metformin dosage in elderly type 2 diabetic patients. Basic Clin Pharmacol Toxicol 2019;124:298-302.

20. Stevens LA, Schmid CH, Greene T, Li L, Beck GJ, Joffe MM, et al. Factors other than glomerular filtration rate affect serum cystatin C levels. Kidney Int 2009;75:652-60.

21. Chin PK, Chew-Harris JS, Florkowski CM, Begg EJ. The performance of contemporary cystatin C-based GFR equations in predicting gentamicin clearance. $\mathrm{Br} J$ Clin Pharmacol 2015;79:268-77.

Supplementary Material: The online version of this article offers supplementary material (https://doi.org/10.1515/labmed-2019-0124). 